\title{
Layer intermixing during metal/metal oxide adsorption: Ti/sapphire(0001)
}

\author{
C. Verdozzi, ${ }^{1}$ P.A. Schultz, ${ }^{2}$ Ruqian Wu, ${ }^{1,3}$ A.H. Edwards, ${ }^{4}$ and Nicholas Kioussis ${ }^{1}$ \\ ${ }^{1}$ Department of Physics, California State University Nortridge, Nortridge California 91330 \\ ${ }^{2}$ Sandia National Laboratories, Albuquerque, New Mexico 87185-1413 \\ ${ }^{3}$ Department of Physics and Astronomy, University of California, Irvine California 92697-4575 \\ ${ }^{4}$ Air Force Research Laboratory, Kirtland AFB, New Mexico 87117-5776
}

(Received 5 July 2002; published 26 September 2002)

\begin{abstract}
First principles density functional calculations for adsorption of $\mathrm{Ti}$ on $\mathrm{Al}_{2} \mathrm{O}_{3}(0001)$ indicate that Ti: $\mathrm{Al}_{2} \mathrm{O}_{3}(0001)$ interfaces become intermixed. Substitutional Ti replaces a surface Al atom rather than a subsurface $\mathrm{Al}$, and the Al-terminated surface is unstable under Ti adsorption. Adsorbed Ti displaces the surface $\mathrm{Al}$, resulting in a mixed Ti/Al interfacial layer instead of a sharp Ti: $\mathrm{Al}_{2} \mathrm{O}_{3}$ interface. Our results provide a coherent picture of the structural and electronic properties of this interface and are consistent with available experimental data.
\end{abstract}

Metal/metal oxide interfaces are crucial to electronics devices, and aluminum $\left(\mathrm{Al}_{2} \mathrm{O}_{3}\right)$ and titanium $\left(\mathrm{TiO}_{2}\right)$ oxides play pivotal roles. ${ }^{1}$ The Ti:sapphire interface at low Ti coverage has notable applications in high power $\operatorname{laser}^{2}$ and optoelectronics waveguide ${ }^{3}$ technologies. More generally, Ti/Al oxide interfaces occur in several applications. These include, for example, Ti/Al alloys for aerospace/automotive applications, when an $\mathrm{Al}_{2} \mathrm{O}_{3}$ coat evolves into $\mathrm{Al}_{2} \mathrm{O}_{3}+\mathrm{TiO}_{2},{ }^{4}$ or nanolayered sandwich structures, such as $\mathrm{Cu}, \mathrm{Ag} / \mathrm{Ti} / \mathrm{Al}_{2} \mathrm{O}_{3}$, where $\mathrm{Ti}$ prewetting improves $\mathrm{Cu}$ and $\mathrm{Ag}$ adhesion. ${ }^{5,6}$

While an extensive literature exists in general for clean oxide surfaces and metal- or oxide-oxide interfaces, much less effort has been devoted to understanding the specific Ti:sapphire interface. Although crucial to the technological applications mentioned above, many aspects of such interface are currently uncertain. Furthermore, the current microscopic understanding of the Ti:sapphire interface derives exclusively from experiment, ${ }^{5-10}$ with temperatures in these studies varying from $300 \mathrm{~K}$ (Ref. 8 ) to above $1100 \mathrm{~K}^{7,9}$ In some cases, experimental evidence is inconclusive: for example, RHEED and ion scattering results ${ }^{9}$ for low-dosage, shallow-implant $\mathrm{Ti}$, indicate that $\mathrm{Ti}$ is in the surface layer atop the (subsurface) Al atoms, even though the actual site preference of the substitution is uncertain. Also, this leads to commensurate growth of crystalline Ti. ${ }^{9}$ Moreover, XPS (Ref. 7) and Auger ${ }^{8}$ data show clearly that at elevated temperatures the metallic $\mathrm{Al} 2 p$ signal is present and the shape of the valence bands, which have primarily oxygen character, are broadened. Furthermore, the Ti $2 p$ signal is shifted. All of this is consistent with the formation of an oxidized $\mathrm{Ti}$ layer and concomitant partial $\mathrm{Al}_{2} \mathrm{O}_{3}$ reduction.

In this paper we report the first $a b$ initio density functional results to elucidate the nature the Ti:sapphire interface. We find that: (i) substitutional Ti prefers to replace the terminating $\mathrm{Al}$ rather than the subsurface $\mathrm{Al}$ and, additionally, little deformation of the surface occurs in the latter case; (ii) a single $\mathrm{Ti}$ adatom on sapphire displaces the terminating Al rather than adsorbing atop the surface; and (iii) a mixed $\mathrm{Ti}_{2} \mathrm{Al}$ adlayer on a Ti-terminated oxide surface is preferred over a sharp $\mathrm{Ti}_{3}: \mathrm{Al}_{2} \mathrm{O}_{3}$ interface. These results indicate that the Al-terminated surface is unstable to Ti adsorption, and predict a mixed $\mathrm{Al} / \mathrm{Ti}$ interfacial layer.

The calculations were performed using density functional theory in the local density approximation, ${ }^{11,12}$ which yields excellent structural energetics for many oxides and specifically for $\mathrm{Al}_{2} \mathrm{O}_{3}$. We used SEQQUEST, ${ }^{13}$ a Gaussian-based linear combination of atomic orbitals method. Standard (nonlocal) norm-conserving pseudopotentials were used for $\mathrm{O}$ (Ref. 14) and Al. ${ }^{15}$ For Ti, the pseudopotential ${ }^{15}$ placed the six $3 p$ (together with the $3 d$ and $4 s$ ) electrons in the valence space, leaving the $3 s$ electrons in the core, and invoked a nonlinear core correction. ${ }^{16}$ The basis sets were high-quality, optimized "double zeta plus polarization" contracted Gaussian functions. ${ }^{17}$

Our calculations involved $1 \times 1$ and $\sqrt{3} \times \sqrt{3}$ surface unit cells (SUC) of sapphire (0001), $a=4.757 \AA$, with slabs nine O-layers thick: $1 \times 1=45,45+1$, and $45+3$ atoms/cell, and $\sqrt{3} \times \sqrt{3}=135$ atoms/cell. Several theoretical ${ }^{18-32}$ and experimental $^{33}$ studies of the sapphire (0001) surface are in broad agreement, showing large Al relaxations deep below the surface and predicting a Al-terminated clean sapphire (0001) surface for UHV environments. This is the surface termination used as a starting point in our calculations. Selected results for the $1 \times 1$ systems were compared to and found consistent with 18 layer slabs. Ti atoms were adsorbed on only one side, and artificial interslab dipole interactions were exactly removed with the local moment counter charge method. ${ }^{34}$ A converged Brillouin zone (BZ) sampling used a hexagonal $k$-grid with 32 (8) points in half of the BZ for the $1 \times 1(\sqrt{3} \times \sqrt{3})$ super-cell, reduced to 12 (4) special $k$-points in the irreducible BZ when threefold symmetry was available. The entire slab geometry was relaxed until the maximum force was $<0.01 \mathrm{eV} / \AA$.

Figure 1 summarizes the structures investigated. Let $\mathrm{Al}_{t}$ represent the bare, Al-terminated, sapphire (0001) surface, depicted in the side and top views in Figs. 1(a) and 1(b,c) respectively. The three different aluminum sites are denoted by $\mathrm{Al1}-\mathrm{Al} 3$, and the two oxygen sites $\mathrm{O} 1-\mathrm{O} 2$ (in order of increasing depth). A Ti-terminated surface in which the terminating $\mathrm{Al}$ is replaced by a $\mathrm{Ti}$ is denoted $\mathrm{Ti}_{t}$. At 


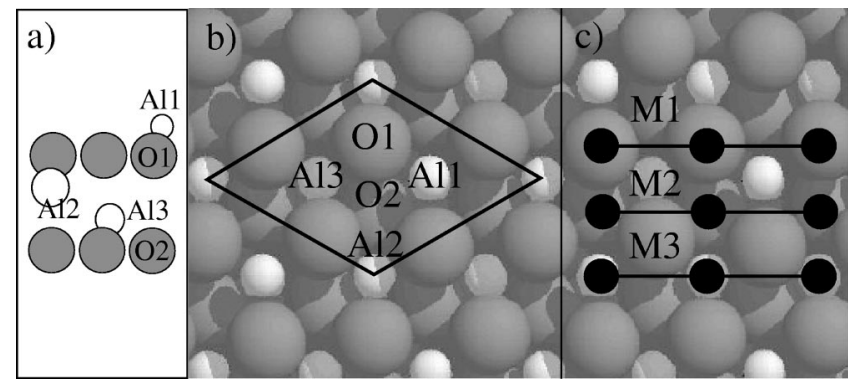

FIG. 1. Side (a) and top (b,c) views of the sapphire (0001) surface. White (gray) circles denote $\mathrm{Al}(\mathrm{O})$ atoms. The $1 \times 1 \mathrm{SUC}$ is shown in (b), and the three in-registry (three-atom) adlayer conformations are depicted in (c).

higher metal coverage $(+3$ atoms), there are three "inregistry" conformations, denoted M1-M3 in Fig. 1(c). For M1(M2), all three adatoms start at equivalent $\mathrm{O} 1(\mathrm{O} 2)$ sites. The M3 conformation occupies the three inequivalent A1 sites, leading to three distinct permutations of atoms for the $\mathrm{Ti}_{2} \mathrm{Al} \mathrm{M} 3$ adlayer considered later. In all cases, the adatoms can relax off of these nonsymmetry sites.

Substitutional Ti at and below the surface. Because the substitutional surface is crucial to understanding the physics of $\mathrm{Ti}$ coverage and intermixing, we first studied whether $\mathrm{Ti}$ preferred to substitute for the surface (A11) or subsurface (A12 or A13) aluminum atoms.

We used a $\sqrt{3} \times \sqrt{3}$ SUC, replacing one of the three surface (Al1) or subsurface (A12) atoms in the SUC with a Ti. The threefold symmetry that exists for these configurations was retained. The Ti-Ti separation of $8.24 \AA$ in this cell is reasonably sufficient to simulate single impurity doping. ${ }^{35} \mathrm{Ti}$ substituting for Al1 induces only small changes in the host; displacements of neighboring $\mathrm{O}$ and $\mathrm{Al}$ are negligible. The Ti sits $0.58 \AA$ above the O1-plane, $0.48 \AA$ higher than the $\mathrm{Al}$ it replaces in $\mathrm{Al}_{t}$, due to its larger size. The minimal structural changes suggest that Ti primarily compensates the local polarity of the replaced Al. As expected, the subsurface (A12) substitution causes somewhat larger host modifications, but the relaxations are still minor and limited to adjacent $\mathrm{Al}$ and $\mathrm{O}$ atoms. This points to a surface "resiliency" as found in experiments. ${ }^{10}$ The O1-layer rumples with deviations of the $\mathrm{O} z$-coordinate from the average by less than $0.09 \AA$, with similar small in-plane displacements. The average O1-O2 layer spacing $\left(2.13 \AA\right.$ for $\left.\mathrm{Al}_{t}\right)$ increases by $<0.07 \AA$, and the Al1-O layer spacing also increases, by $<0.05 \AA$. Totalenergy results favor Ti replacing the surface Al1 over subsurface Al2 (consistent with experiment ${ }^{9}$ ) by $0.92 \mathrm{eV}$. Removing the Al1 atom and placing Ti above Al3 is not a viable alternative; the energy is $1.8 \mathrm{eV}$ higher.

Additional insight can be gleaned from the local density of states (LDOS) projected onto Löwdin orbitals. In Fig. 2(a) [2(b)] we show the LDOS for the surface (subsurface) $\mathrm{Ti}$ impurity, and for the neighboring $\mathrm{O}$ atoms.

The integrated LDOS, Löwdin populations (LP), of the next-nearest neighbor $\mathrm{O}$ atoms $(6.66 e)$ already resembles that in clean $\mathrm{Al}_{t}(6.68 e$ ) (for neutral $\mathrm{O}, \mathrm{LP}=6.0$ ). The $\mathrm{Ti}$ impurity LDOS has a peak at $\mathrm{E}_{F}$, which is larger and narrower for the subsurface $\mathrm{Ti}$ [Fig. 2(b)] than for the Ti in the

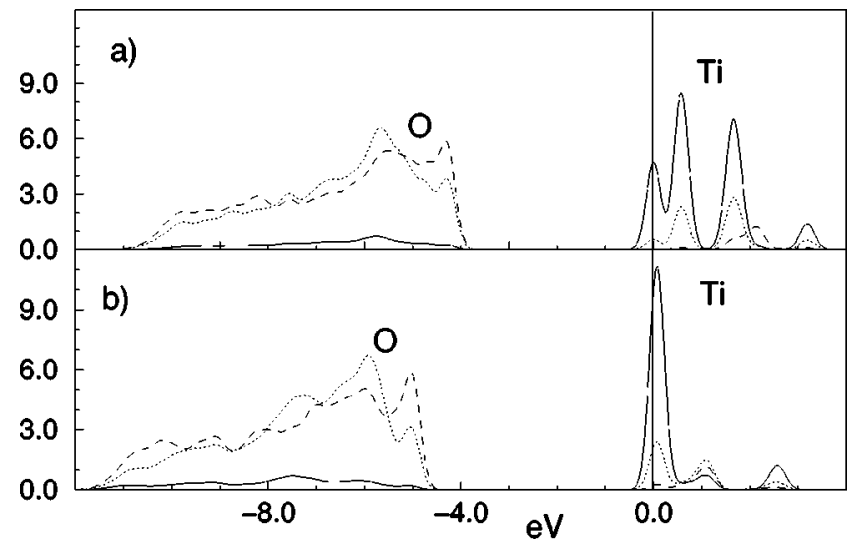

FIG. 2. LDOS for Ti impurities in (a) surface-Al1 and (b) subsurface-A12 sites. Dotted (dashed) curves, magnified four times, are LDOS for the nearest-neighbor (next nearest) $\mathrm{O}$ to the Ti. In both panels, solid curves refer to $\mathrm{Ti}$, and $\mathrm{E}_{F}=0$.

terminating surface site. The surface Ti $d$-states are strongly split, with an excess of 0.4 electrons of $3 z^{2}-r^{2}$ character. In contrast, the subsurface $\mathrm{Ti}$ has a more localized, compact electron population with more nearly constant $d$-LP's in response to the more isotropic Madelung field.

Isolated $\mathrm{Al}(\mathrm{Ti})$ atom on the $T i_{t}\left(A l_{t}\right)$ surface. Having established that a substitutional Ti prefers to replace the terminating $\mathrm{Al}$, we next examine whether a single $\mathrm{Ti}$ adatom added to a clean $\mathrm{Al}_{t}$ surface adsorbs atop the surface $\left(\mathrm{Ti}: \mathrm{Al}_{t}\right)$, or displaces a surface $\mathrm{Al}$, forming $\mathrm{Al}: \mathrm{Ti}_{t}$. The $0 \mathrm{~K}$ calculations reported here should give insight into the interface formation $^{36}$ and diffusion barriers. However, a comprehensive theoretical description would require the inclusion of kinetic effects and explicit calculations of transition barrier energies, beyond the scope of this work.

The ground state configuration is found within those associated with $\mathrm{Al}: \mathrm{Ti}_{t}$, with $\mathrm{Al}$ relaxing into an adatom site between $\mathrm{O} 1$ and $\mathrm{O} 2$ [Fig. 1(a)]. The $\mathrm{Al}$ relaxes to this site from either the $\mathrm{O} 1$ or $\mathrm{O} 2$ starting point. Total energies, referenced to this state, of five distinct adatom sites [i.e., Al1-3 and $\mathrm{O} 1-2$, Fig. 1(a)] for both $\mathrm{Al}: \mathrm{Ti}_{t}$ and $\mathrm{Ti}: \mathrm{Al}_{t}$ are listed in Table I. The ground state is favored by $0.40 \mathrm{eV}$ over the next most stable configuration: $\mathrm{Ti}: \mathrm{Al}_{t}$ with $\mathrm{Ti}$ atop the $\mathrm{Al} 3$ site. In the presence of the $\mathrm{Ti}$ adatom at the $\mathrm{Al} 3$ site the surface $\mathrm{Al}$ atom moves $0.77 \AA$ outwards from its clean surface position [Al1 in Fig. 1(b)]. Concomitant with this relaxation, the Al LP changes from $1.87 e$ on the clean surface to $2.41 e$, closer to the neutral value of $3.0 e$. The $\mathrm{O}$ ionicity remains nearly constant, leading to a smaller Coulombic attraction between the surface aluminum and its neighboring $\mathrm{O}$ ions. This is consistent with the fact that $\mathrm{Ti}$ is less electronegative than Al. Although the difference is small, the net change in ionicity may result from a feedback wherein the $\mathrm{Al}$ atom loses more charge by separating further from its neighbors. We expect similar behavior whenever the metallic adatom has a smaller electronegativity than $\mathrm{Al}^{37}$

When comparing $\mathrm{Al}: \mathrm{Ti}_{t}$ to $\mathrm{Ti}_{\mathrm{Al}} \mathrm{Al}_{t}$ we see the same qualitative trend; even though the partial s-, p-, d-LPs for the surface atoms are rather different, the total LP for the Ti and surface $\mathrm{O}$ are virtually identical, while the LP difference for 
TABLE I. Relative energies (in eV) for relaxed surface configurations started from different initial starting points, for the $\mathrm{Al}: \mathrm{Ti}_{t}$ and $\mathrm{Ti}: \mathrm{Al}_{t}$ single adatom surfaces, and for the three-atom adlayer $\mathrm{Ti}_{3}: \mathrm{Al}_{t}$ and $\mathrm{Ti}_{2} \mathrm{Al}: \mathrm{Ti}_{t}$ geometries. Energies in bold indicate constrained threefold symmetry and $u$ denotes structurally unstable configurations. Energies marked by an asterisk pertain to extrema (local maxima or saddle points) rather than minima: forces are zero, but a very small perturbation would lead to downhill motion to one of the other configurations. The (normal) distance (in $\AA$ ) of the terminating metal atom to the O1-plane is given for both the clean $\mathrm{Ti}_{t}$ and $\mathrm{Al}_{t}\left(d_{\text {clean }}\right)$, and for the minimum energy configuration with adsorbates $\left(d_{\text {adsorb }}\right)$.

\begin{tabular}{lccccccc}
\hline \hline & $\mathrm{O} 1$ & $\mathrm{O} 2$ & $\mathrm{~A} 11$ & $\mathrm{~A} 12$ & $\mathrm{Al} 3$ & $d_{\text {clean }}$ & $d_{\text {adsorb }}$ \\
\hline $\mathrm{Al}: \mathrm{Ti}_{t}$ & 0 & 0 & $\mathbf{1 . 1 5}$ & $\mathbf{0 . 6 7}$ & $\mathbf{0 . 4 5}$ & 0.58 & 0.67 \\
$\mathrm{Ti}_{\mathrm{Al}} t$ & 1.20 & 1.20 & $\mathbf{4 . 6 5}$ & $\mathbf{1 . 2 0}$ & $\mathbf{0 . 4 0}$ & 0.10 & 0.87 \\
& & & $\mathrm{M} 3$ & $\mathrm{M} 3$ & $\mathrm{M} 3$ & & \\
& $\mathrm{M} 1$ & $\mathrm{M} 2$ & $\mathrm{~A} 11$ & $\mathrm{~A} 2$ & $\mathrm{~A} 13$ & $d_{\text {clean }}$ & $d_{\text {adsorb }}$ \\
\hline $\mathrm{Ti}_{2} \mathrm{Al}_{\mathrm{Ti}} \mathrm{Ti}_{t}$ & 1.27 & $u$ & 0.35 & 0.0 & $u$ & 0.58 & 0.61 \\
$\mathrm{Ti}_{3}: \mathrm{Al}_{t}$ & $\mathbf{2 . 4 4}$ & $\mathbf{4 . 3 1 *}$ & $\mathbf{1 . 4 6}$ & $\cdots$ & $\cdots$ & 0.10 & 0.77 \\
\hline \hline
\end{tabular}

the surface $\mathrm{Al}$ atom differs by only $\sim 0.2$ electron. The similar LP's and the small total energy difference $(0.40 \mathrm{eV})$, suggest that $\mathrm{Ti}$ displacing $\mathrm{Al}$ in the surface results from a subtle charge redistribution.

The Ti:Al ${ }_{t}$ configuration assumed in Ref. 9 is partially unsupported by our calculation, since Ti largely prefers Al3 to $\mathrm{Al} 2$ adsorption sites. The $\mathrm{O} 1$ and $\mathrm{O} 2$ adatom sites are not even metastable in $\mathrm{Ti}_{\mathrm{Al}} \mathrm{Al}_{t}$ : starting from either of these positions, a Ti adatom relaxes to the Al2 site. The Ti position in $\mathrm{Al}: \mathrm{Ti}_{t}$ is mostly unchanged by the $\mathrm{Al}$ adatom. The calculated Ti adsorption energy is $\sim 5.0 \mathrm{eV}$, substantial, but not atypical for sapphire surfaces. Previous results ${ }^{22}$ for $1 / 3$ ML Pt on the clean surface reported an adsorption energy of $2.9 \mathrm{eV}$. The LDOS results for $\mathrm{Al}: \mathrm{Ti}_{t}\left(\mathrm{Ti}_{\mathrm{Al}}\right)$ are shown in Fig. 3(a) [3(b)]. The three key atoms are the surface $\mathrm{Al}$ and $\mathrm{Ti}$, and their nearest neighbor, the intermediate $\mathrm{O}$. The most important feature is the behavior of $\mathrm{Ti}$ and $\mathrm{Al}$ LDOS close to $E_{F}$. In Ti: $\mathrm{Al}_{t}$ [Fig. 3(b)], the Ti LDOS exhibits a large peak across $E_{F}$, with minimal overlap with the Al LDOS. The situation is quite different in $\mathrm{Al}^{-\mathrm{Ti}_{t}}[\mathrm{Fig}$. 3(a)]; the change in the local environment reflects into a redistribution of states near $\mathrm{E}_{f}$; specifically, a splitting in the Ti LDOS at $E_{F}$ transfers significant intensity $0.4 \mathrm{eV}$ below $E_{F}$ that overlaps strongly with the $\mathrm{Al}$ LDOS.

$\mathrm{Ti}_{3}\left(\mathrm{Ti}_{2} \mathrm{Al}\right)$ onto the $A l_{t}\left(\mathrm{Ti}_{t}\right)$ surface. The final issue we consider is whether higher coverages of Ti on sapphire(0001)

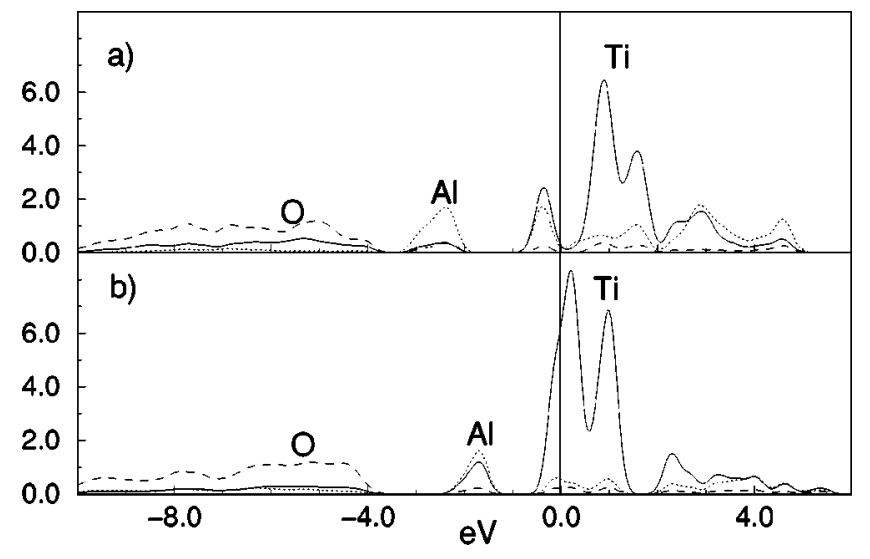

FIG. 3. LDOS for (a) $\mathrm{Al}: \mathrm{Ti}_{t}$ and (b) $\mathrm{Ti}_{\mathrm{Al}} \mathrm{Al}_{t}$. Dotted, dashed, and solid curves refer to $\mathrm{Al}, \mathrm{O}$, and $\mathrm{Ti}$, respectively, and $\mathrm{E}_{F}=0$.

leave an oxidized $\mathrm{Ti}$ in the surface (with a mixed metal overlayer), or segregate into a distinct Ti-overlayer. The results are presented in the last two rows of Table I, and show a mixed $\mathrm{Ti}_{2} \mathrm{Al}$ overlayer (atop metal sites, $\mathrm{Al}$ in the $\mathrm{Al} 2$ site) is favored by $1.46 \mathrm{eV}$ over the lowest-energy $\mathrm{Ti}_{3}$ overlayer configuration (atop $\mathrm{Al}$ positions). Both the $\mathrm{Ti}_{3}$ and the $\mathrm{Ti}_{2} \mathrm{Al}$ overlayers exhibit considerable buckling, also found for other metals on sapphire. The $\mathrm{Ti}_{3}$ adlayer has the same effect on the sapphire substrate that a single Ti adatom has (see above), moving the terminating $\mathrm{Al}$ outwards from its clean surface position. This suggests that the same mechanism of electron redistribution and structural rearrangement applies. A discussion of the electronic properties will be presented elsewhere.

Our study is the first $a b$ initio description of the $\mathrm{Ti}_{x} \mathrm{Al}_{2} \mathrm{O}_{3}$ interface and provides a theoretical rationalization, consistent with available experiments, of many aspects of the interfacial behavior. These are the surface rigidity of sapphire for dilute shallow Ti impurities; the Ti preference for surface rather than subsurface replacement of $\mathrm{Al}$; the instability of the $\mathrm{Al}$ terminated sapphire surface in the presence of Ti; and the consequent formation of a mixed $\mathrm{Al} / \mathrm{Ti}$ layer at the onset of interface formation. These results should be germane to other $\mathrm{Ti}_{x} \mathrm{Al}_{y} \mathrm{O}_{z}$ phases of Ti/Al-based technological materials, and informative to the adsorption of other metals on the $\mathrm{Al}_{2} \mathrm{O}_{3}$ surface.

C.V., R.W., and N.K. acknowledge support from the Parsons and Keck Foundations. R.W. also acknowledges the DOE-BES support (Grant No. DE-FG03-99ER14948). Sandia is a multiprogram laboratory operated by Sandia Corporation, a Lockheed Martin Company, for the United States Department of Energy, under Contract. No. DEAC0494AL85000.

\footnotetext{
${ }^{1}$ The Surface Science of Metal Oxides, edited by V. E. Henrich and P. A. Cox (Cambridge University Press, Cambridge, 1994); see also contributions to the volume "The Surface Science of Metal Oxides," Faraday Discuss. 114 (1999). For a theoretical review, see C. Noguera, Surf. Rev. Lett. 8, 121 (2001).

${ }^{2}$ D.M. Pennington, C.G. Brown, T.E. Cowan, S.P. Hatchett, E.
}

Henry, S. Herman, M. Kartz, M. Key, J. Koch, A.J. MacKinnon, M.D. Perry, T.W. Phillips, M. Roth, T. C Sangster, M. Singh, R.A. Snavely, M. Stoyer, B.C. Stuart, and S.C. Wilks, IEEE J. Quantum Electron. 6, 676 (2000).

${ }^{3}$ P. Manoravi, P.R. Willmott, J.R. Huber, Jr., and T. Greber, Appl. Phys. A: Mater. Sci. Process. 69, S865 (1999). 
${ }^{4}$ E.H. Copland, B. Gleeson, and D.J. Young, Acta Mater. 47, 2937 (1999)

${ }^{5}$ G. Dehm, C. Scheu, M. Ruhle, and R. Raj, Acta Mater. 46, 759 (1998).

${ }^{6}$ R. Lazzari and J. Jupille, Surf. Sci. 482-485, 823 (2001).

${ }^{7}$ H.J. Kang, C.H. Kim, N.S. Park, and M.W. Kim, Appl. Surf. Sci. 101, 160 (1996).

${ }^{8}$ S. Bernath, T. Wagner, S. Hofmann, and M. Ruhle, Surf. Sci. 400, 335 (1998).

${ }^{9}$ T. Suzuki, S. Hishita, K. Oyoshi, and R. Souda, Surf. Sci. 437, 289 (1999).

${ }^{10}$ H. Lee, S.M. Lee, E.T. Ada, B. Kim, M. Weiss, S.S. Perry, and J.W. Rabalais, Nucl. Instrum. Methods Phys. Res. B 157, 226 (1999)

${ }^{11}$ J.P. Perdew and A. Zunger, Phys. Rev. B 23, 5048 (1981).

${ }^{12}$ D.M. Ceperley and B.J. Alder, Phys. Rev. Lett. 45, 566 (1980).

${ }^{13}$ P. A. Schultz (unpublished). A description of the method is in P.J. Feibelman, Phys. Rev. B 35, 2626 (1987).

${ }^{14}$ N. Troullier and J.L. Martins, Phys. Rev. B 43, 1993 (1991).

${ }^{15}$ D.R. Hamann, Phys. Rev. B 40, 2980 (1989).

${ }^{16}$ S.G. Louie, S. Froyen, and M.L. Cohen, Phys. Rev. B 26, 1738 (1982).

${ }^{17}$ P.A. Schultz, unpublished (available by request: paschul @ sandia.gov).

${ }^{18}$ J. Guo, D.E. Ellis, and D.J. Lam, Phys. Rev. B 45, 3204 (1992).

${ }^{19}$ I. Manassidis, A. De Vita, and M.J. Gillan, Surf. Sci. Lett. 285, L517 (1993); Philos. Mag. B 69, 879 (1994).

${ }^{20}$ T.J. Godin and J.P. LaFemina, Phys. Rev. B 49, 7691 (1994).

${ }^{21}$ C. Kruse, M.W. Finnis, J.S. Lin, M.C. Payne, V.Y. Milman, A. DeVita, and M.J. Gillan, Philos. Mag. Lett. 73, 377 (1996).
${ }^{22}$ C. Verdozzi, D.R. Jennison, P.A. Schultz, and M.P. Sears, Phys. Rev. Lett. 82, 799 (1999).

${ }^{23}$ R. Di Felice and J.E. Northrup, Phys. Rev. B 60, R16 287 (1999).

${ }^{24}$ X.G. Wang, A. Chaka, and M. Scheffler, Phys. Rev. Lett. 84, 3650 (2000).

${ }^{25}$ J.R.B. Gomes, I.D.R. Moreira, P. Reinhardt, A. Wander, B.G. Searle, N.M. Harrison, and F. Illas, Chem. Phys. Lett. 341, 412 (2001).

${ }^{26}$ K.C. Hass, W.F. Schneider, A. Curioni, and W. Andreoni, Science 282, 265 (1998).

${ }^{27}$ P.J. Eng, T.P. Trainor, G.E. Brown, G.A. Waychunas, M. Newville, S.R. Sutton, and M.L. Rivers, Science 288, 1029 (2000).

${ }^{28}$ I.G. Batirev, A. Alavi, M.W. Finnis, and T. Deutsch, Phys. Rev. Lett. 82, 1510 (1999).

${ }^{29}$ W. Zhang and J.R. Smith, Phys. Rev. Lett. 85, 3225 (2000).

${ }^{30}$ Q.S. Ma, K. Klier, H.S. Cheng, J.W. Mitchell, and K.S. Hayes, J. Phys. Chem. B 105, 2212 (2001).

${ }^{31}$ E.A. Jarvis and E.A. Carter, J. Phys. Chem. B 105, 4045 (2001).

${ }^{32}$ Z. Lodziana and J.K. Nørskov, J. Chem. Phys. 115, 11261 (2001).

${ }^{33}$ See for example, C.F. Walters, K.F. McCarthy, E.A. Soares, and M.A. Van Hove, Surf. Sci. 464, L732 (2000).

${ }^{34}$ P.A. Schultz, Phys. Rev. B 60, 1551 (1999).

${ }^{35}$ C. Verdozzi, D.R. Jennison, P.A. Schultz, M.P. Sears, J.C. Barbour, and B. G Potter, Phys. Rev. Lett. 80, 5615 (1998).

${ }^{36}$ Y.F. Zhukovskii, E. A Kotomin, P.W.M. Jacobs, and A.M. Stoneham, Phys. Rev. Lett. 84, 1256 (2000).

${ }^{37}$ A similar behavior was explicitly noted in Refs. 21,29,32 for $\mathrm{Nb}$, $\mathrm{Ag}$, and $\mathrm{Pd}$, respectively. 